\title{
NEW TESTS OF PERTURBATIVE QCD INSPIRED BY HYPOTHETICAL TAU LEPTONS ${ }^{a}$
}

\author{
J. R. PELAEZ ${ }^{1}$, S.J. BRODSKY ${ }^{2}$ and N. TOUMBAS ${ }^{2}$ \\ ${ }^{1}$ Departamento de Física Teórica. Universidad Complutense de Madrid. 28040 Madrid. SPAIN \\ ${ }^{2}$ Stanford Linear Accelerator Center. Stanford University, Stanford, California 94309. U.S.A.
}

Inspired by the relation between the hadronic decay of the $\tau$ lepton and the $e^{+} e^{-}$annihilation into hadrons, we derive new tests of perturbative QCD. We design a set of commensurate scale relations to test the self-consistency of leading-twist QCD predictions for any observable which defines an effective charge. This method provides renormalization scheme and scale invariant probes of $\mathrm{QCD}$ which can be applied over wide data ranges.

Talk presented at the XXXIVth Rencontres de Moriond:

QCD and High Energy Hadronic Interactions

Les Arcs, Bourg St. Maurice, France, March 20-27th, 1999

\section{Introduction}

The $\tau$ lepton hadronic width, $R_{\tau}=\Gamma\left(\tau^{-} \rightarrow \nu_{\tau}+\right.$ hadrons $) / \Gamma\left(\tau^{-} \rightarrow \nu_{\tau} e^{-} \overline{\nu_{e}}\right)$ plays an important role in the determination of the QCD coupling ${ }^{1}$. Its analysis has been performed using integral moments which minimize the sensitivity to the low energy data ${ }^{2}$. In particular, just by integrating the measured spectral functions up $M$ we can simulate the physics of hypothetical $\tau$ leptons $^{2}$ with masses $M$ smaller than the physical one. Their hadronic widths yield a crucial test of perturbative QCD (PQCD), since they are related to the $e^{+} e^{-}$annihilation cross section into hadrons $R_{e^{+} e^{-}}$through

$$
R_{\tau}(M)=\frac{2}{\left(\sum_{f} q_{f}^{2}\right)} \times \int_{0}^{M^{2}} \frac{d s}{M^{2}}\left(1-\frac{s}{M^{2}}\right)^{2}\left(1+\frac{2 s}{M^{2}}\right) R_{e^{+} e^{-}}(\sqrt{s}) .
$$

In this paper we report on a recent proposal ${ }^{3}$ of self-consistency tests of PQCD, motivated by the above relations, which can be applied to any observable which defines an effective charge. These tests are examples of relations between observables at two different scales, which are called "commensurate scale relations" 4 .

Effective charges are defined as the entire radiative contribution to an observable ${ }^{5}$. For instance, assuming $f$ massless flavors, we have

$$
R_{e^{+} e^{-}}(\sqrt{s}) \equiv\left(3 \sum_{f} q_{f}^{2}\right)\left[1+\frac{\alpha_{R}(\sqrt{s})}{\pi}\right] \quad, \quad R_{\tau}(M) \equiv R_{\tau}^{0}(M)\left[1+\frac{\alpha_{\tau}(M)}{\pi}\right],
$$

where the effective charges $\alpha_{R}$ and $\alpha_{\tau}$ can be written as a series in $\alpha_{s} / \pi$ in any given renormalization scheme. Their relevance is given by the fact that they satisfy the renormalization group equation with the same coefficients $\beta_{0}$ and $\beta_{1}$ as the usual coupling $\alpha_{s}$.

At this point we can make use of the Mean Value Theorem in eq.(1), to relate $\alpha_{R}$ and $\alpha_{\tau}$ by a scale shift

$$
\alpha_{\tau}(M)=\alpha_{R}\left(\sqrt{s^{*}}\right), \quad \text { with } \quad \lambda_{\tau}=\frac{\sqrt{s^{*}}}{M}=\exp \left[-\frac{19}{24}-\frac{169}{128} \frac{\alpha_{R}(M)}{\pi}+\cdots\right]
$$

${ }^{a}$ Research partially supported by the Spanish CICYT under contract AEN97-1693 and the U.S. Department of Energy DE-AC03-76SF00515. 
where the value of $\lambda_{\tau}$ is a prediction of $N L O$ leading twist $Q C D$. This result was first obtained in ${ }^{4}$ by using NNLO, however, we will see how it is due to the fact that both effective charges evolve with universal $\beta_{0}$ and $\beta_{1}$ coefficients. ${ }^{3}$

\section{Tests of PQCD for a general observable}

These relations can be generalized to arbitrary observables $O(s)$, with an associated effective charge $\alpha_{O}$, by defining new effective charges

$$
\alpha_{f}(M) \equiv \frac{\int_{0}^{M^{2}} \frac{d s}{M^{2}} f\left(\frac{s}{M^{2}}\right) \alpha_{O}(\sqrt{s})}{\int_{0}^{M^{2}} \frac{d s}{M^{2}} f\left(\frac{s}{M^{2}}\right)},
$$

where we can choose $f(x)$ to be any smooth, integrable function of $x=s / M^{2}$. Once more

$$
\alpha_{f}(M)=\alpha_{O}\left(\sqrt{s_{f}^{*}}\right), \quad 0 \leq s_{f}^{*} \leq M^{2} .
$$

Note that this relation only involves data for the observable $O(s)$ and thus provides a selfconsistency test for the applicability of leading twist QCD. To obtain the relation between the commensurate scales, we consider the running of $\alpha_{O}$ up to third order

$$
\frac{\alpha_{O}(\sqrt{s})}{\pi}=\frac{\alpha_{O}(M)}{\pi}-\frac{\beta_{0}}{4} \ln \left(\frac{s}{M^{2}}\right)\left(\frac{\alpha_{O}(M)}{\pi}\right)^{2}+\frac{1}{16}\left[\beta_{0}^{2} \ln ^{2}\left(\frac{s}{M^{2}}\right)-\beta_{1} \ln \left(\frac{s}{M^{2}}\right)\right]\left(\frac{\alpha_{O}(M)}{\pi}\right)^{3} \ldots
$$

We substitute for $\alpha_{O}$ in eq. (4) to find

$$
\frac{\alpha_{f}(M)}{\pi}=\frac{\alpha_{O}(M)}{\pi}-\frac{\beta_{0}}{4}\left(\frac{I_{1}}{I_{0}}\right)\left(\frac{\alpha_{0}(M)}{\pi}\right)^{2}+\frac{1}{16}\left[\beta_{0}^{2}\left(\frac{I_{2}}{I_{0}}\right)-\beta_{1}\left(\frac{I_{1}}{I_{0}}\right)\right]\left(\frac{\alpha_{O}(M)}{\pi}\right)^{3} \ldots
$$

where $I_{l}=\int_{0}^{1} f(x)(\ln x)^{l} d x$. Hence

$$
\lambda_{f} \equiv \frac{\sqrt{s_{f}^{*}}}{M}=\exp \left[\frac{I_{1}}{2 I_{0}}+\frac{\beta_{0}}{8}\left(\left(\frac{I_{1}}{I_{0}}\right)^{2}-\frac{I_{2}}{I_{0}}\right) \frac{\alpha_{O}(M)}{\pi}\right] .
$$

Note that $\lambda_{f}$ is constant to leading order, and therefore $\alpha_{f}$ satisfies the same renormalization group equation as $\alpha_{O}$ with the same coefficients $\beta_{0}$ and $\beta_{1}$; i.e., $\alpha_{f}$ is an effective charge.

Note that eq.(5) relates an observable with an integral over itself. It is also possible to obtain differential relations ${ }^{6}$, but here we will simply illustrate the use of the integral relations.

\section{Example: self-consistency test of $R_{e^{+} e^{-}}$data.}

Let us then set $O=R_{e^{+} e^{-}}$. In order to suppress the low energy region, where non-perturbative effects are important, we shall set $f(x)=x^{k}$, with $k$ some positive number. Thus

$$
\alpha_{k}(M)=\alpha_{R}\left(\lambda_{k} M\right) \quad \text { with } \quad \lambda_{k}=e^{\frac{-1}{2(1+k)}}
$$

When comparing with $R_{e^{+} e^{-}}$data, we take into account the mass effects using ${ }^{7}$ :

$$
\begin{aligned}
R_{e^{+} e^{-}}(\sqrt{s}) & =3 \sum_{1}^{f} q_{i}^{2} \frac{v_{i}\left(3-v_{i}^{2}\right)}{2}\left[1+g\left(v_{i}\right) \frac{\alpha_{R}(\sqrt{s})}{\pi}\right] \equiv R_{0}(\sqrt{s})+R_{S c h}(\sqrt{s}) \frac{\alpha_{R}(\sqrt{s})}{\pi} \\
g(v) & =\frac{4 \pi}{3}\left[\frac{\pi}{2 v}-\frac{3+v}{4}\left(\frac{\pi}{2}-\frac{3}{4 \pi}\right)\right]
\end{aligned}
$$



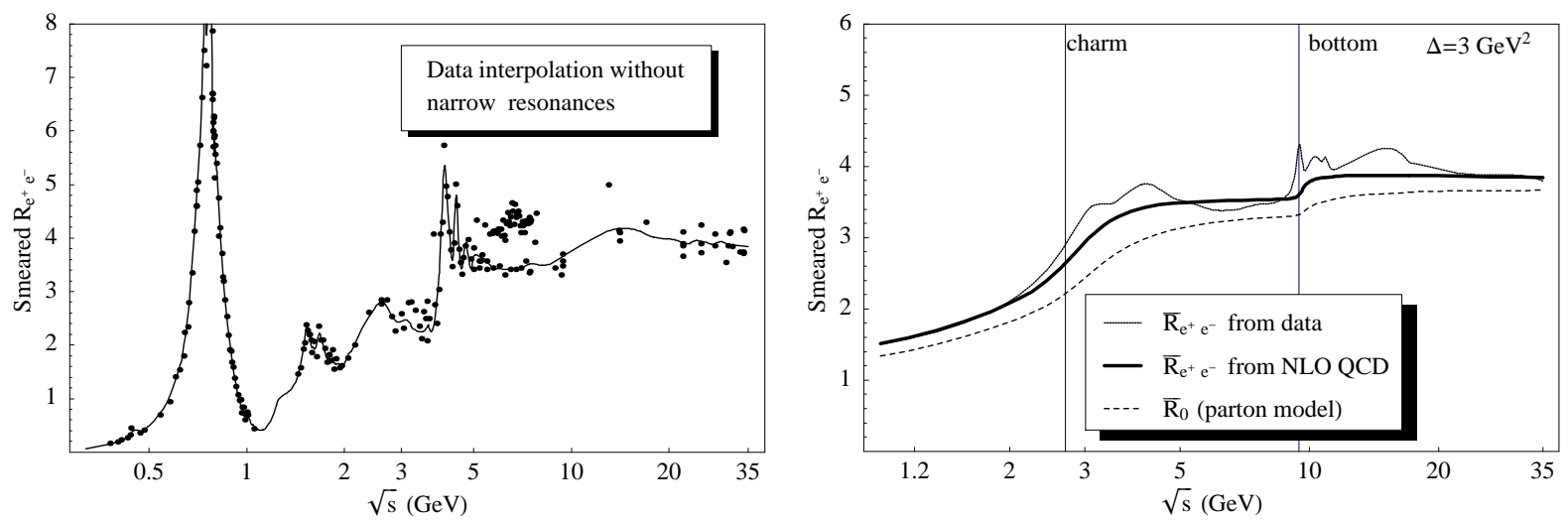

Figure 1.a) Interpolation of the central values of $R_{e}+_{e^{-}}$data (see ${ }^{3}$ for references). Note the discrepancy in the central values of experiments between 5 and $10 \mathrm{GeV}$. b) Smeared $R_{e^{+} e^{-}}$.

where $v_{i}$ is the velocity of the initial quarks in their CM frame. The $v_{i}\left(3-v_{i}^{2}\right) / 2$ factor is the parton model mass dependence and $g(v)$ is a QCD modification of the Schwinger correction. The quark masses have been taken as effective parameters which provide a good fit to the smeared data. All these corrections spoil eq.(9), but they are only important near the quark thresholds. At higher energies they tend to unity and quark masses become irrelevant, which is why our study is restricted to this regime. Still we cannot compare directly with the data since we observe hadrons, not quarks. Following ${ }^{7}$ we define smeared quantities as follows:

$$
\bar{R}(\sqrt{s})=\frac{\Delta}{\pi} \int_{0}^{\infty} \frac{R\left(\sqrt{s^{\prime}}\right)}{\left(s-s^{\prime}\right)^{2}-\Delta^{2}} d s^{\prime}
$$

By smearing $R_{e^{+} e^{-}}$over a range of energy, $\Delta E$, we focus the physics to the time $\Delta t=1 / \Delta E$ where an analysis in terms of quarks and gluons is appropriate. In what follows we use the standard value $\Delta=3 \mathrm{GeV}^{27,8}$. The smearing effect can be seen comparing Fig.1.a, which shows an interpolation of the $R_{e^{+} e^{-}}$data, (see ${ }^{3}$ for references) with Fig. 1.b. Note that any fit using the QCD functional dependence will always satisfy eq.(9) identically. To avoid this bias, we have parameterized the narrow resonances using their Breit-Wigner form, and we have interpolated the remaining data ( see $^{3}$ for details).

Finally, using eqs.(10) and (12), we define smeared charges:

$$
\bar{\alpha}_{R}(\sqrt{s})=\frac{\bar{R}_{e^{+} e^{-}}(\sqrt{s})-\bar{R}_{0}(\sqrt{s})}{\bar{R}_{S c h}(\sqrt{s})},
$$

and similarly for $\bar{\alpha}_{k}$. According to the previous discussion we expect the smeared charges to satisfy eq.(8) in energy regions where the threshold corrections can be neglected.

Thus, in Fig.2.a we compare $\bar{\alpha}_{R}\left(\sqrt{s^{*}}\right)$ with $\bar{\alpha}_{k}\left(\sqrt{s^{*}} / \lambda_{k}\right)$. The agreement for $\alpha_{0}$ is poor since the low energy region is not suppressed enough. However we find a reasonable agreement for $\alpha_{1}$ in several regions, agreement which disappears if we do not shift the scales. There are two regions of particular interest where we find a disagreement: First, from 5 to $10 \mathrm{GeV}$ where there is a well known incompatibility between experiments (see Fig.1.a and ref. ${ }^{9}$ ). In Fig.1.a. we have kept the most recent data, as it is standard in the literature, but still their central values are systematically lower than the QCD predictions. which is why eq.(9) does not seem to hold. Our test correctly shows this incompatibility.

Second, we show in Fig. 2.b., the physical $\tau$ region where, considering that we are using LO QCD and central data values, the agreement looks quite satisfactory. This is encouraging for the applicability of PQCD in the region near the real $\tau$ lepton. However, at energies $\sqrt{s} \sim 1.5 \mathrm{GeV}$, our results support the claims that the $R_{e^{+} e^{-}}$data could be $6-7 \%$ lower than expected from $R_{\tau}$ data $^{10}$. Note, however, that our conclusions have been obtained using only data on $R_{e^{+} e^{-}}$. 

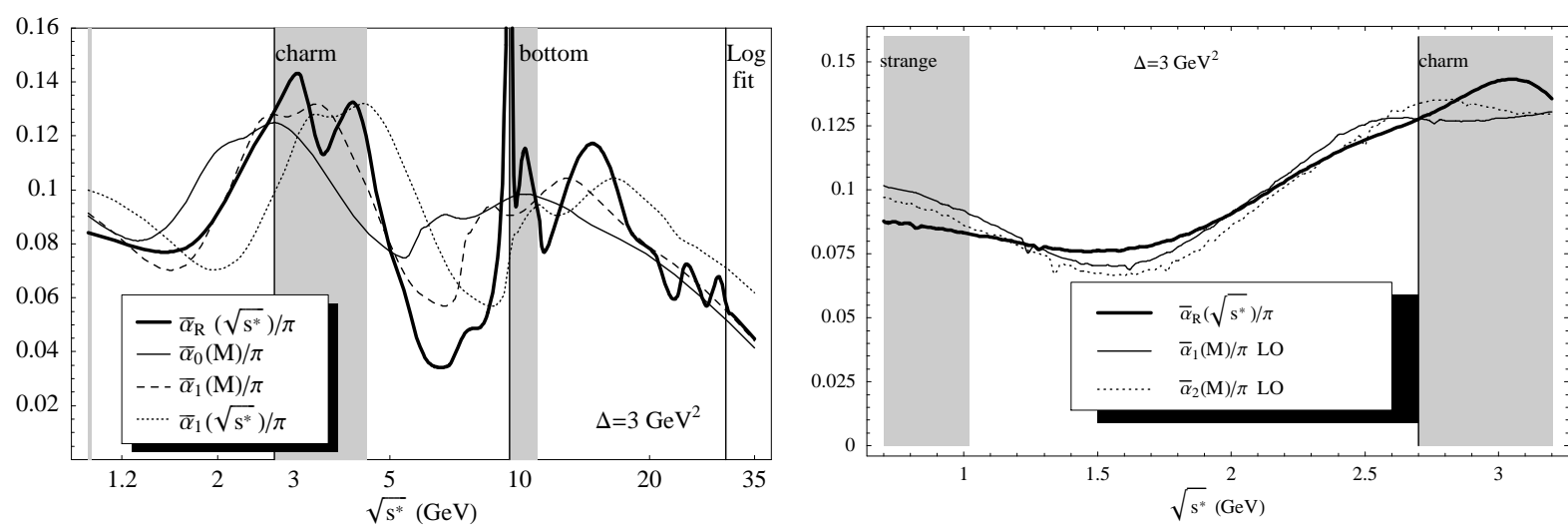

Figure 2.a) Comparison between $\bar{\alpha}_{R}\left(\sqrt{s^{*}}\right)$ and different $\bar{\alpha}_{k}$ moments at $M=\sqrt{s^{*}} / \lambda_{k}$. The dotted line shows how the agreement is spoilt if we do not shift $\sqrt{s^{*}}$ to $M$. b) Comparison between $\bar{\alpha}_{R}\left(\sqrt{s^{*}}\right)$ and different $\bar{\alpha}_{k}$ moments at $M=\sqrt{s^{*}} / \lambda_{k}$ in the low energy region.

\section{Conclusions}

Motivated by the relation between $R_{e^{+} e^{-}}$and the $R_{\tau}$, as well as the ideas of commensurate scale relations, we have presented new tests of PQCD. They can be applied in a wide energy range to any observable which defines an effective charge, and they are renormalization scheme and scale independent.

As an example, we have tested the self-consistency of existing $R_{e^{+} e^{-}}$data according to PQCD. We have found a good agreement in the real $\tau$ region but incompatibilities around the $1.5 \mathrm{GeV}$ region and in the range of 5 to $10 \mathrm{GeV}$, supporting previous claims obtained by different methods. The advantage of our approach is that it only relates the observable with itself, which can be very useful when applied to other experiments.

\section{Acknowledgments}

Research partially supported by the Spanish CICYT under contract AEN97-1693 and the U.S. Department of Energy DE-AC03-76SF00515. J.R.P. thanks the SLAC theory group for their kind hospitality.

\section{References}

1. E. Braaten, Phys. Rev. Lett. 60 (1988), Phys. Rev. D39 1458 (1989); E. Braaten, S. Narison and A. Pich, Nucl. Phys. B373 581 (1992).

2. F. Le Diberder and A. Pich, Phys. Lett. B289 165 (1992); S. Narison and A. Pich, Phys. Lett. B304 359 (1993), S. Narison, Phys. Lett. B358 113 (1995) and Phys. Lett. B361 121 (1995), M. Girone and M. Neubert, Phys. Rev. Lett. 763061 (1996); S. Groote, J. G. Korner and A. A. Pivovarov, Phys. Lett. B407 66 (1997); M. Davier, hep-ph/9802447; R. Barate et al. Eur. Phys. J. C4 409 (1998). K. Ackerstaff et al., hep-ex/9808019.

3. S. J. Brodsky, J. R. Peláez and N. Toumbas, slac-pub-7976, hep-ph/ 9810424. To appear in Phys.Rev.D.

4. S. J. Brodsky and H. J. Lu Phys. Rev. D51 3652 (1995).

5. G. Grunberg, Phys. Rev. D29 2315 (1984).

6. S. J. Brodsky, C. Merino and J. R. Peláez. slac-pub-8033.

7. E. C. Poggio, H. R. Quinn and S. Weinberg, Phys. Rev. D13 1958 (1976). T. W. Appelquist and H. D. Politzer, Phys. Rev. Lett. 34, 43 (1975); Phys. Rev. D12 1404 (1975). J. Schwinger, Particles, Sources and Fields, Vol.II, Addison-Wesley, New York, 1973.

8. A. C. Mattingly and P.M. Stevenson, Phys. Rev. D49 437 (1994).

9. M. L. Swartz, Phys. Rev. D53 5268 (1996).

10. S. Groote et al., Phys. Rev. Lett. 792763 (1997). 